teratoma was derived from totipotential cells and was always fused with the spinal cord. The cells of later inclusion gave rise to more superficial and simple tumours, whilst those included at the very last stage in neural tube closure gave rise to dermal sinuses. He then discussed the nature of iatrogenic epidermoid tumours following lumbar puncture.

He described a series of four teratomas, four dermoids, and four dermal sinuses. He noted that all the dermoid tumours occurred in adults of blood group A. Low back or sciatic pain was the commonest presentation and progressive paraparesis was common in the dermoids. Changes in the plain radiographs were found in five cases and included spina bifida, scalloping of the posterior aspects of vertebral bodies, widened interpedicular widths, and calcified intervertebral discs. A short history was not incompatible with a long-standing tumour. The commonest site was in the region of the conus medullaris lying dorsal to the cord.

Operative treatment was satisfactory in all cases and follow-up of one to 11 years had not shown any evidence of recurrence.

He concluded that even partial removal could produce good results, children in particular making a rapid recovery. Dermoid cysts, though simple histologically, produced the worst disability and had a worse prognosis.

\section{ELECTRONIC DATA PROCESSING IN THE SPINA BIFIDA PROBLEM}

B. H. DAwson (Salford) discussed the problems concerning the methods and results of surgical repair of the spina bifida baby. He instanced many factors that could influence the prognosis, such as the site, size, and situation of the lesion and the amount of epithelial covering. The time after birth of referral and presence of infection were also important.

He said that assessments of functional and intellectual capacities in these patients in the long term was difficult. Some succumbed in the first few months of life; some survived with gross neurological defects. In others there were serious problems of orthopaedic and urological nature.

Experience with marginal punch card analysis of the data showed that this technique had a very limited value in handling such a multitude of prognostic factors. Efforts using the 80-column 10-row punch card variety of computer tabulation were described.

The stages of computer analysis were described and the scope and limitations of computer handling of biomedical data were discussed in general terms. Discussion of the detailed results of this data analysis was reserved for subsequent meetings. 